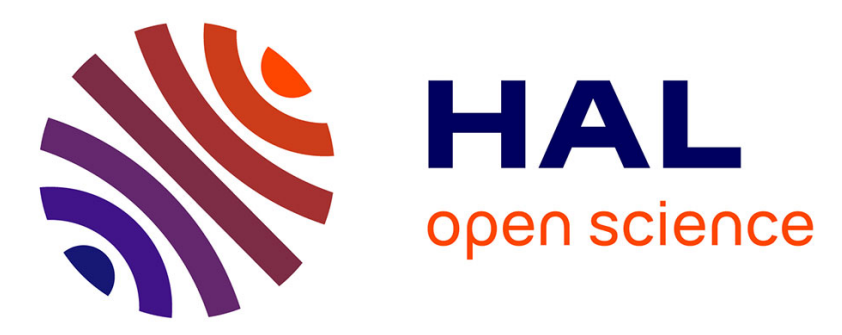

\title{
A multi-modal approach using a non-parametric model to extract fetal ECG
}

\author{
Saman Noorzadeh, Bertrand Rivet, Pierre-Yves Guméry
}

\section{To cite this version:}

Saman Noorzadeh, Bertrand Rivet, Pierre-Yves Guméry. A multi-modal approach using a nonparametric model to extract fetal ECG. ICASSP 2015 - 40th IEEE International Conference on Acoustics, Speech and Signal Processing, Apr 2015, Brisbane, Australia. 10.1109/ICASSP.2015.7178086 . hal-01208418

\section{HAL Id: hal-01208418 \\ https://hal.science/hal-01208418}

Submitted on 2 Oct 2015

HAL is a multi-disciplinary open access archive for the deposit and dissemination of scientific research documents, whether they are published or not. The documents may come from teaching and research institutions in France or abroad, or from public or private research centers.
L'archive ouverte pluridisciplinaire HAL, est destinée au dépôt et à la diffusion de documents scientifiques de niveau recherche, publiés ou non, émanant des établissements d'enseignement et de recherche français ou étrangers, des laboratoires publics ou privés. 


\title{
A MULTI-MODAL APPROACH USING A NON-PARAMETRIC MODEL TO EXTRACT FETAL ECG
}

\author{
Saman Noorzadeh ${ }^{1,2}$, Bertrand Rivet ${ }^{1}$, Pierre-Yves Guméry ${ }^{2}$ \\ ${ }^{1}$ GIPSA-lab, CNRS UMR-5216, Grenoble University, Grenoble, France \\ 2 TIMC-lab, CNRS UMR-5525, Joseph Fourier University, Grenoble,France
}

\begin{abstract}
This study presents a non-parametric method to extract and separate maternal and fetal Electrocardiogram (ECG) from an abdominal channel. The proposed method relies on the use of two additional reference signals related to the maternal and to the fetal ECGs. The abdominal recording fetal and maternal ECGs are modeled from their respective references using a Gaussian process. Both of these signals are modeled considering a thoracic ECG reference, and an abdominal PCG reference, respectively for maternal ECG and fetal ECG. This general method provides a good behavior to extract fetal ECG as stated by the numerical experiments and shows that it can use only 1-bit reference signals, which can be easier to record and more efficient to process.
\end{abstract}

Index Terms - fetal ECG, non-parametric model, Gaussian process, multi-modal, PCG, multi-channel approach

\section{INTRODUCTION}

While monitoring fetal electrocardiogram (ECG) during pregnancy can provide important clinical information about the health of the fetus [1], the ECG recorded from the abdomen does not directly provide the fetal ECG. This abdominal channel is contaminated by several noise disturbances; however, the main noise contribution is the maternal ECG which is even stronger than fetal ECG in this data channel.

Different methods have been proposed to separate maternal and fetal ECG which use different schemes in signal processing. Among these methods, some are proposed to use a single channel recording of data including kalman filter [2], and non-parametric model [3], and other methods are based on multi-channel data like singular value decomposition $[4,5]$, blind source separation [6, 7], and adaptive filters $[8,9,10]$.

Although the latter methods like Independent Component Analysis (ICA), and adaptive filters are efficient approaches, they use several number of data channels which make the method costly considering the calculation time of processing the channels. For example in ICA [6], for maternal ECG subspace 3 components are needed, and 2 for fetal ECG subspace are at least needed, so the number of observation channels should be greater than 5 channels. Also in (linear) adaptive filters [8] at least one reference signal is used to separate fetal ECG from other noises, i.e. in [9] two chest leads are used to denoise fetal ECG.

In the present study, we propose a multi-channel method which uses a non-parametric approach using Gaussian process to model the ECG signal. The separation of maternal and fetal ECGs would be possible by modeling each of these signals. This method needs one reference data channel for each ECG that we intend to model. We will then show that these reference signals can be only 1-bit signals which are easy to record by cheap sensors, and a 1-bit ADC and are also more memory-efficient and has less time complexity to process.

Through this study we will present a multi-modal approach using 2 different signals: ECG and PCG (Phonocardiogram) to model the ECG of the fetus and will show how multi-modality [11] can help to have complementary information about the ECG covariance function, although the modalities are of two different types: ECG is the electrical activity of the heart, while PCG is the audio signal caused by the closure of valves of the heart [12]. A synchronous record of ECG and PCG is shown in Fig 1.
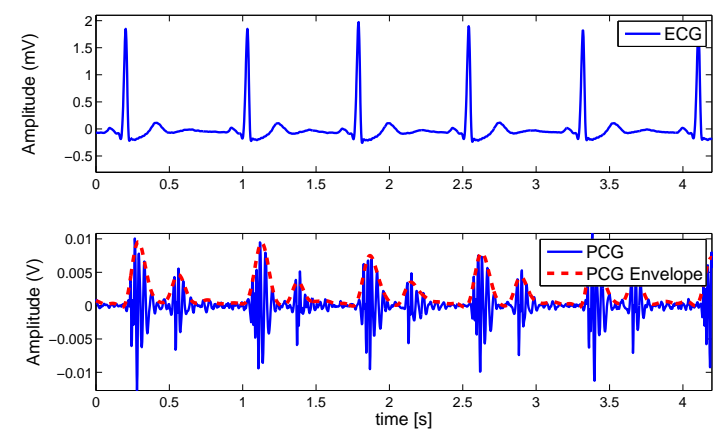

Fig. 1: Synchronous ECG and PCG.

In the rest of the paper, we first explain the Gaussian process used to model an ECG signal using a reference signal channel in Section 2.1, and then the problem of fetal ECG extraction using this method and a multi-modal approach will 
be presented in Section 2.2. The results are then compared in Section 3, and the conclusions are presented in Section 4.

\section{MULTI-MODAL METHOD}

The aim of this section is to provide a non-parametric multimodal method to model an ECG using a reference signal. The first part details the modeling and denoising an ECG signal having one reference channel, and the second part extends it to use two reference signals to model maternal and fetal ECGs and to extract them from an abdominal noisy ECG channel.

\subsection{Modeling ECG Using Gaussian Process}

A non-parametric model of ECG is to describe it as a Gaussian process (GP) [13], specified by its second order statistics: mean function $m(t)$ and covariance function $k\left(t, t^{\prime}\right)$. Considering ECG as a real process, we define mean and covariance functions as

$$
\begin{aligned}
m(t) & =\mathbb{E}[E C G(t)], \\
k\left(t, t^{\prime}\right) & =\mathbb{E}\left[(E C G(t)-m(t))\left(E C G\left(t^{\prime}\right)-m\left(t^{\prime}\right)\right)\right],
\end{aligned}
$$

where $\mathbb{E}[\cdot]$ is the expectation operator, and the GP is noted as:

$$
E C G(t) \sim \mathcal{G P}\left(m(t), k\left(t, t^{\prime}\right)\right)
$$

The mean function is usually defined as 0 for notional simplicity. The covariance function plays a crucial role in describing the Gaussian process, since it determines the characteristics of sample functions drawn from the GP distribution. Due to physiological variabilities, ECG is a quasi-periodic signal, so the covariance function should reflect this property. However, the usually-used covariance functions cannot precisely describe it: one way is thus to use a two-step model: a strictly periodic covariance function and a nonlinear time wrapping to adjust the quasi-periodicity as described in [3]. To overcome this difficulty, a schema to take advantage of another reference signal to define the covariance function is proposed in this study.

Consider a noisy ECG signal, noted $y(t)$ as the observation, which contains the desired ECG, $s(t)$, and noise, $n(t)$. Having another reference channel, which can be of another kind (e.g., PCG), called $x(t)$, we can take advantage of it to define the covariance function to model $s(t)$, if there is a dependence between $s(t)$ and $s_{x}(t)$

$$
\begin{aligned}
& y(t)=s(t)+n(t), \\
& x(t)=s_{x}(t)+n_{x}(t),
\end{aligned}
$$

where $n_{x}(t)$ is the noise of the reference signal. Considering that $s_{x}(t)$ depends on $s(t)$ and that noises are independent from each other and from sources, we can also assume the dependency of our desired signal, $s(t)$, and the reference signal, $x(t)$.
Let's define $\vec{v}_{T}(t)=[v(t), v(t-1), \cdots, v(t-T)]$ for any signal $v(t) . s(t)$ depends on $\vec{x}_{N}(t)$ in the GP scheme, and $\vec{x}_{N}(t)$ is the set of inputs of GP such that:

$$
s(t)=f\left(\vec{x}_{N}(t)\right)+\epsilon(t),
$$

where $\epsilon(t)$ is the additive noise, and the $f(\cdot)$ function is described by a GP:

$$
f\left(\vec{x}_{N}(t)\right) \sim \mathcal{G P}\left(0, k\left(\vec{x}_{N}(t), \vec{x}_{N}\left(t^{\prime}\right)\right)\right) .
$$

The proposed covariance function is defined by

$$
\begin{aligned}
& k\left(\vec{x}_{N}(t), \vec{x}_{N}\left(t^{\prime}\right)\right)= \\
& \sigma^{2} \exp \left(-\frac{\left(\vec{x}_{N}(t)-\vec{x}_{N}\left(t^{\prime}\right)\right)^{T}\left(\vec{x}_{N}(t)-\vec{x}_{N}\left(t^{\prime}\right)\right)}{2 l^{2}}\right)
\end{aligned}
$$

with the hyper-parameters $\sigma, l$, and $N$. This covariance function defines a good assumption of the observation signal, using a window of $N$ samples of $x(t)$ signal. $\sigma$ is to model the amplitude of the signal, and $l$ as the length-scale models the smoothness of the signal. A GP model of noise can be also defined as equation (6)

$$
\begin{aligned}
n(t) & \sim \mathcal{G P}\left(0, k_{n}\left(t, t^{\prime}\right)\right), \\
k_{n}\left(t, t^{\prime}\right) & =\sigma_{n} \delta\left(t-t^{\prime}\right),
\end{aligned}
$$

where the hyper-parameter $\sigma_{n}$ models the noise amplitude and $\delta($.$) is the delta Dirac function.$

Considering the covariance functions for ECG and noise, as defined in equations (5) and (6), the ECG signal $s(t)$ that we intend to denoise can then be estimated according to

$$
\hat{s}\left(t_{*}\right)=\mathbf{k}\left(t_{*}\right) \mathbf{K}^{-1} \mathbf{y},
$$

where $\mathbf{y}=\left[y\left(t_{1}\right), \cdots, y\left(t_{n}\right)\right]^{T}$, and $n$ is the length of the signal, and

$$
\mathbf{k}\left(t_{*}\right)=\left[k\left(\vec{x}_{N}\left(t_{*}\right), \vec{x}_{N}\left(t_{1}\right)\right), \cdots, k\left(\vec{x}_{N}\left(t_{*}\right), \vec{x}_{N}\left(t_{n}\right)\right)\right],
$$

and $\mathbf{K}$ is the covariance matrix of the observation signal whose $(\mathrm{i}, \mathrm{j})$ th element is defined as

$$
K_{i, j}=k\left(\vec{x}_{N}\left(t_{i}\right), \vec{x}_{N}\left(t_{j}\right)\right)+k_{n}\left(t_{i}, t_{j}\right) .
$$

It is worth noting that the proposed method does not specify the nature of the reference signal: this latter one can be of the same kind (i.e. ECG) or of any other kind (e.g., PCG) as soon as it is correlated to the modeled ECG. Moreover, it is also possible to replace the full reference channel, noted $x(t)$ here, with a 1-bit signal, let's say $x_{q}(t)$. Since the assumption of dependence of the desired signal, $s(t)$, and $x_{q}(t)$ is still correct, we can use only a 1-bit reference signal to propose a cheaper device. It should also be mentioned that despite adaptive filters that use linear filters for estimation, the explained non-parametric method provides a non-linear estimation using a GP. It should be noted that the dependency we refered to in equation (3) does not assume a linear relationship between the input and the output [14]. 


\subsection{Fetal ECG Extraction using Multi-Modality}

The abdominal signal channel, $s_{a}(t)$, contains three main signals: the maternal ECG $\left(s_{m}(t)\right)$, fetal ECG $\left(s_{f}(t)\right)$, and noise $(n(t))$ :

$$
s_{a}(t)=s_{m}(t)+s_{f}(t)+n(t) .
$$

As explained before, for each of these signals we have to consider one reference signal. The reference signal for modeling maternal ECG is an ECG data recorded from the chest, $x(t)$; and the reference for the fetal ECG is the envelope of a PCG signal which is recorded from the abdomen to be referred to as the fetal PCG. This reference signal is noted as $p(t)$,

Maternal ECG $s_{m}(t)$ can be modeled, according to the proposed model, using a chest ECG reference $x(t)$ as

$$
\begin{aligned}
s_{m}(t) & =f\left(\vec{x}_{N}(t)\right)+\epsilon_{m}(t), \\
f\left(\vec{x}_{N}(t)\right) & \sim \mathcal{G P}\left(0, k_{m}\left(\vec{x}_{N}(t), \vec{x}_{N}\left(t^{\prime}\right)\right)\right),
\end{aligned}
$$

with $k_{m}(\cdot, \cdot)$ defined by (5). By a GP model we can express the fetal ECG to be dependent on the PCG envelope:

$$
s_{f}(t)=f\left(\vec{p}_{M}(t)\right)+\epsilon_{f}(t),
$$

with

$$
f\left(\vec{p}_{M}(t)\right) \sim \mathcal{G P}\left(0, k_{f}\left(\vec{p}_{M}(t), \vec{p}_{M}\left(t^{\prime}\right)\right)\right),
$$

where the covariance function, $k_{f}(\cdot, \cdot)$ is defined by (5).

Considering the covariance functions used for ECGs (5) and for noise (6), the hyper-parameters for maternal ECG, fetal ECG and noise are defined as $\theta_{m}=\left\{\sigma_{m}, l_{m}, N\right\}, \theta_{f}=$ $\left\{\sigma_{f}, l_{f}, M\right\}$, and $\theta_{n}=\left\{\sigma_{n}\right\}$, respectively. These hyperparameters need to be learned. In the maximum likelihood framework, they are estimated by maximizing the log likelihood

$$
\log p\left(\mathbf{s}_{\mathbf{a}} \mid \boldsymbol{\theta}\right)=-\frac{1}{2} \log (2 \pi)-\frac{1}{2} \log (|\mathbf{K}|)-\frac{1}{2} \mathbf{s}_{\mathbf{a}}{ }^{T} \mathbf{K}^{-1} \mathbf{s}_{\mathbf{a}},
$$

where $\mathbf{s}_{a}=\left[s_{a}\left(t_{1}\right), \cdots, s_{a}\left(t_{n}\right)\right]^{T}$ with the length of $n$ samples, and $\mathbf{K}$ is the covariance matrix of the abdominal data whose $(i, j)$ th entry is defined as

$K_{i, j}=k_{m}\left(\vec{x}_{N}\left(t_{i}\right), \vec{x}_{N}\left(t_{j}\right)\right)+k_{f}\left(\vec{p}_{M}\left(t_{i}\right), \vec{p}_{M}\left(t_{j}\right)\right)+k_{n}\left(t_{i}, t_{j}\right)$.

The fetal ECG can then be estimated from the following equation.

$$
\hat{s}_{f}\left(t_{*}\right)=\mathbf{k}_{\mathbf{f}}\left(t_{*}\right) \mathbf{K}^{-1} \mathbf{s}_{\mathbf{a}},
$$

with

$$
\mathbf{k}_{f}\left(t_{*}\right)=\left[k_{f}\left(\vec{p}_{M}\left(t_{*}\right), \vec{p}_{M}\left(t_{1}\right)\right), \cdots, k_{f}\left(\vec{p}_{M}\left(t_{*}\right), \vec{p}_{M}\left(t_{n}\right)\right)\right] \text {. }
$$

It is necessary to point out that both references used for maternal and fetal ECG estimations can be replaced by 1-bit signals.

\section{RESULTS}

In this section, first the use of one and two reference signal channels are compared using synthetic data, and then the results of fetal ECG extraction from a real data is shown.

Considering the observation signal of equation (7), we intend to estimate fetal ECG, $s_{f}(t)$. Two approaches can be used here: using only one reference signal to model $s_{m}(t)$ [15], or using two references to model both $s_{m}(t)$ and $s_{f}(t)$. The synthetic data for maternal and fetal ECGs and noise are summed, to form the synthetic abdominal ECG, defined as $s_{a}(t)$. The two mentioned approaches are then tested to extract fetal ECG. In the former approach we use a synthetic thoracic ECG channel, noted as $x(t)$ in the previous section, related to maternal contribution. In the second approach beside using the maternal reference, we also use a synthetic PCG, noted as $p(t)$, to model fetal ECG. The quadratic errors of estimated fetal ECGs are shown in red in Fig. 2. According to this figure, the performance is better in the second approach when considering two reference signals. We have then replaced the references of both approaches with 1-bit signals, and the error results are depicted in blue in Fig. 2. It is clear that using 1-bit references instead of full references does not degrade the performance.

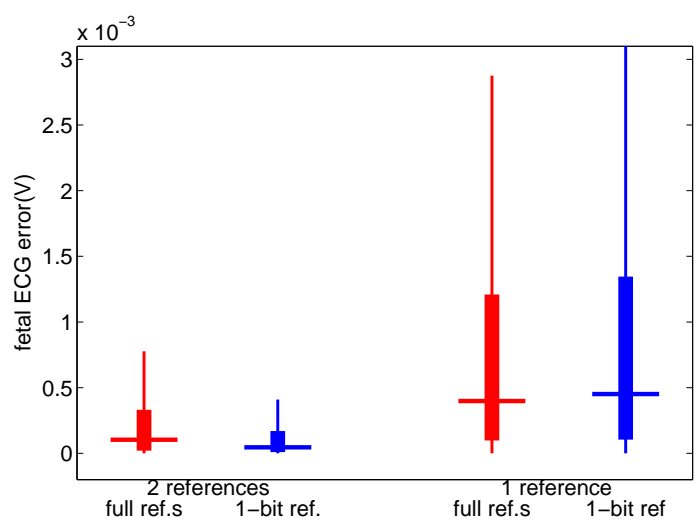

Fig. 2: The error of estimated fetal ECG.

We have also tested the method on the real data. The data used here is recorded from a pregnant woman in the 8th month of pregnancy. One ECG electrode is placed on the abdomen to record the noisy channel of data as a mixture of maternal and fetal ECG. The abdominal PCG is also recorded using a condenser microphone device and the thoracic ECG is also recorded. The recorded ECGs are filtered between $0.1 \mathrm{~Hz}$ and $50 \mathrm{~Hz}$ and the fetal PCG signal is filtered between $30 \mathrm{~Hz}$ and $100 \mathrm{~Hz}$. To obtain the envelope of the PCG, we used the Hilbert transform with a moving average filter [16]. Fig. 3 shows the extraction of maternal and fetal ECGs from the 


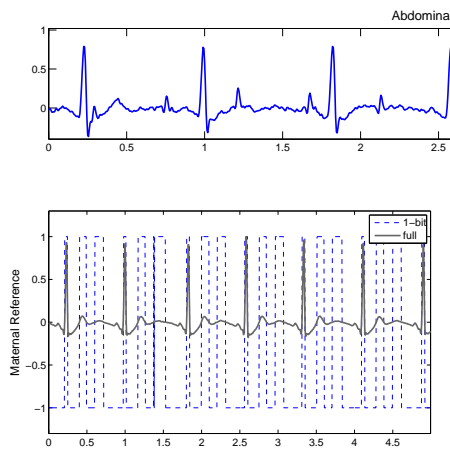

\section{Adominal ECG}
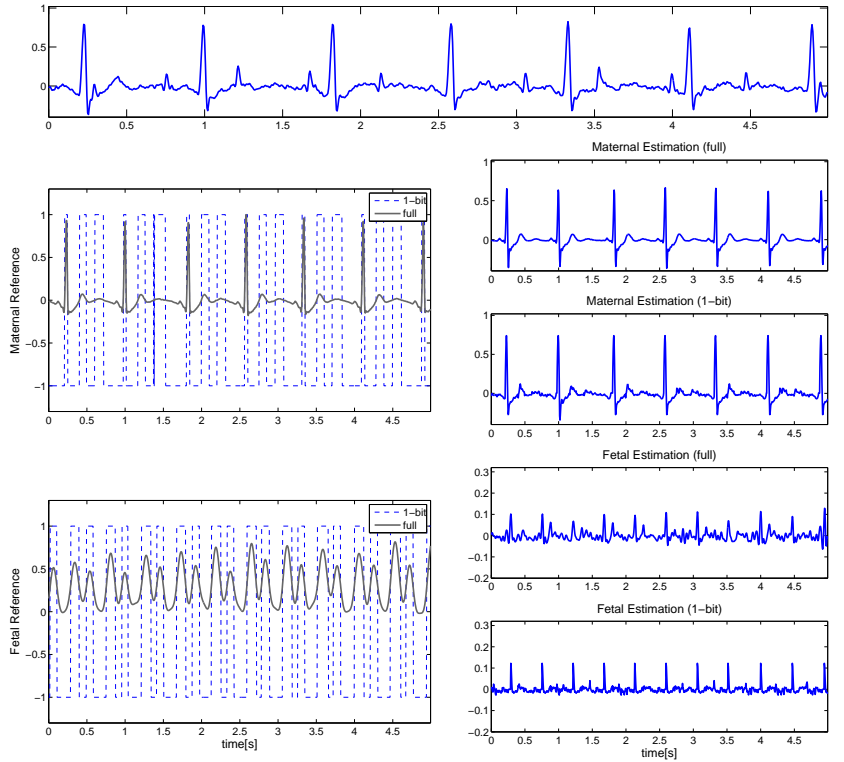

Fig. 3: Maternal and fetal ECG extraction using full and 1-bit references. The maternal and fetal estimation on top are results of using full references.

noisy abdominal channel using the thoracic maternal ECG as the reference to model the maternal ECG, and the envelope of fetal PCG as the reference to extract the fetal ECG. Both of these references are also replaced by their 1-bit version, and the results of extraction are shown in this figure. As shown here, both full and 1-bit references are able to extract the fetal ECG; however, the fetal ECG estimated is less noisy with the 1-bit reference.

\section{CONCLUSIONS}

Through this work we have presented a multi-channel method to extract fetal ECG from the noisy abdominal channel. This method is based on non-parametric models, and we have used Gaussian process to model the ECG signals.

The abdominal ECG is mainly a mixture of maternal and fetal ECGs. Each of these ECG signals can be modeled using a reference data. The reference data is used to define the covariance function of the ECG signal that we intend to model. More specifically, the covariance function can be defined properly using a window of the reference signal which is used as the input of the Gaussian process. The reference signal to model maternal ECG can be a record of ECG data recorded from the chest, and to model fetal ECG, the abdominal PCG, considered as fetal PCG, can play the role of the reference signal.
This method is a multi-channel method as the classical methods like adaptive filters; however despite adaptive filters this is a non-linear method. The method also provides a multimodal approach to use the PCG data in modeling fetal ECG. This approach first validates the relationship between these two data, and second it gives better results in extraction of fetal ECG when having a separate model for fetus instead of using only one model for mother, as validated in section 3 . In the same section we have shown that the reference signals can be replaced by 1-bit reference without a noticeable change in the results. Using 1-bit reference signals have several advantages: first of all, they are easier to record with 1-bit ADCs, secondly, they are less memory consuming, and finally they can be processed faster in time.

\section{ACKNOWLEDGMENT}

This work has been supported by the LabEx PERSYVALLab (ANR-11-LABX-0025) and ERC AdG-2012-320864CHESS.

\section{REFERENCES}

[1] J.P. Neilson, "Fetal electrocardiogram (ECG) for fetal monitoring during labour," Cochrane Database Syst Rev, vol. 3, 2006.

[2] R. Sameni, M.B. Shamsollahi, C. Jutten, and G.D. Clifford, "A nonlinear bayesian filtering framework for ecg denoising," Biomedical Engineering, IEEE Transactions on, vol. 54, no. 12, pp. 2172-2185, 2007.

[3] M. Niknazar, B. Rivet, and C. Jutten, "Fetal ECG extraction from a single sensor by a non-parametric modeling," in Signal Processing Conference (EUSIPCO), 2012 Proceedings of the 20th European. IEEE, 2012, pp. 949-953.

[4] P. P. Kanjilal, S. Palit, and G. Saha, "Fetal ECG extraction from single-channel maternal ecg using singular value decomposition," Biomedical Engineering, IEEE Transactions on, vol. 44, no. 1, pp. 51-59, 1997.

[5] D Callaerts, B De Moor, J. Vandewalle, Sansen, G. Vantrappen, and J. Janssens, "Comparison of SVD methods to extract the foetal electrocardiogram from cutaneous electrode signals," Medical and Biological Engineering and Computing, vol. 28, no. 3, pp. 217-224, 1990.

[6] V. Zarzoso, A.K. Nandi, and E. Bacharakis, "Maternal and foetal ecg separation using blind source separation methods," Mathematical Medicine and Biology, vol. 14, no. 3, pp. 207-225, 1997. 
[7] V. Zarzoso and A.K. Nandi, "Noninvasive fetal electrocardiogram extraction: blind separation versus adaptive noise cancellation," Biomedical Engineering, IEEE Transactions on, vol. 48, no. 1, pp. 12-18, 2001.

[8] B. Widrow, J.R. Glover Jr, J.M. McCool, J. Kaunitz, C.S. Williams, R.H. Hearn, J.R. Zeidler, E. Dong Jr, and R.C. Goodlin, "Adaptive noise cancelling: Principles and applications," Proceedings of the IEEE, vol. 63, no. 12, pp. 1692-1716, 1975.

[9] E. R. Ferrara and B. Widrow, "Fetal electrocardiogram enhancement by time-sequenced adaptive filtering," Biomedical Engineering, IEEE Transactions on, , no. 6, pp. 458-460, 1982.

[10] M. Yelderman, B. Widrow, J. M. Cioffi, E. Hesler, and J.A. Leddy, "ECG enhancement by adaptive cancellation of electrosurgical interference," Biomedical Engineering, IEEE Transactions on, , no. 7, pp. 392-398, 1983.

[11] D. Lahat, T. Adali, and C. Jutten, "Challenges in multimodal data fusion," in Proceedings of the 22nd European Signal Processing Conference (EUSIPCO 2014), 2014.

[12] R.M. Rangayyan and R.J. Lehner, "Phonocardiogram signal analysis: a review.," Critical reviews in biomedical engineering, vol. 15, no. 3, pp. 211-236, 1986.

[13] C.E. Rasmussen, "Gaussian processes for machine learning," 2006.

[14] F. Pérez-Cruz, S. Van Vaerenbergh, J. Murillo-Fuentes, M. Lázaro-Gredilla, and I. Santamaria, "Gaussian processes for nonlinear signal processing: An overview of recent advances," Signal Processing Magazine, IEEE, vol. 30, no. 4, pp. 40-50, 2013.

[15] S. Noorzadeh, B. Rivet, and P.Y. Guméry, "Enhancing fetal ECG using gaussian process," in 10th International Conference on Mathematics in Signal Processing, 2014, accepted for publication.

[16] S. Choi and Z. Jiang, "Comparison of envelope extraction algorithms for cardiac sound signal segmentation," Expert Systems with Applications, vol. 34, no. 2, pp. 1056-1069, 2008. 